\title{
Design of a Non-Overlapping Clock Generator for RFID Transponder EEPROM
}

\author{
Labonnah F. Rahman, Mamun B. I. Reaz, and Mohammad Marufuzzaman
}

\begin{abstract}
A non-overlapping clock (NOC) generator circuit is designed for the successful operation of high voltage generator (HVG) implementation in low-power applications like radio frequency identification (RFID) tag EEPROM. The NOC generator has been implemented in $0.18 \mu \mathrm{m}$ CMOS process. The designed NOC can generate two stable anti-phase clock signals as output, which is used in charge pump (CP) circuit with low power dissipation. The NOC generator required lower power dissipation with $359.87 \mathrm{nW}$ under power supply voltage (VDD) $1.8 \mathrm{~V}$. Moreover, this designed NOC generator produced faster clock signals with $0.972 \mu \mathrm{S}$ as the settling time.
\end{abstract}

Index Terms-NOC, HVG, RFID, CP, EEPROM.

\section{INTRODUCTION}

RFID is an identification system, which is used to transfer and receive data from the tag/transponder using a reader through radio frequency. The identification code is attached to an object for tracking. Storing and reading the data without getting in touch with or involving contact between the transponder and reader makes RFID technology a great application. Transponder is a chip attached inside any product, animal, or even a person for the purpose of identification and tracking [1]. The tag contains an electronic microchip, which is fabricated as a low power integrated circuit (IC). Depending on the device functionality, the tag memory may consist of ROM, RAM, non-volatile memory (EEPROM, Flash) and data buffers [2].

Among all the memory types, embedded non-volatile memory (NVM) is the mostly used tag memory. The NVM has received much attention as it can be broadly applied into RFID tag, SOC and FPGA systems, etc. Conversely, the prerequisite of additional masks and fabrication steps makes NVMs such as electrically erasable programmable read only memory (EEPROM) and flash memory are highly expensive than a standard CMOS process. Many researchers took these challenges and developed NVM in a standard CMOS logic process [3]-[8]. They have the advantages of low cost, low power and compatibility with the standard CMOS process. On the other hand, the maintenance and endurance characteristics due to the NMOS tunneling junction or the

Manuscript received February 8, 2014; revised May 16, 2014. This work was supported by the research grant DLP-2013-016 from the Ministry of Science, Technology and Innovation (MOSTI) and Universiti Kebangsaan Malaysia.

Labonnah F. Rahman, Mamun B. I. Reaz, and Mohammad Marufuzzaman are with the Department of Electrical, Electronic and Systems Engineering, Faculty of Engineering and Built Environment Universiti Kebangsaan Malaysia, 43600 UKM Bangi, Malaysia (e-mail: labonnah.deep@gmail.com, marufsust@gmail.com). single ended memory cell architecture with a too thin oxide are unsatisfactory [3], [5]. It has large area/bit and consumes much power as each bit cell includes its own high voltage switch [4], [7]. To generate these high voltages, an internal HVG circuit is required [9]. A HVG circuit generates the higher voltage inside the EEPROM during the erase operation. Approximately $10 \mathrm{M} / \mathrm{V}$ is generated across the thin oxide between the floating gate and the $\mathrm{n}+$ source diffusion (or the channel) [10]. Typically, HVG circuit consists of a charge pump (CP) and some auxiliary circuits, including NOC generator circuit, discharge circuit, comparator circuit, and voltage divider circuit.

In analog, as well as digital circuits, clock plays an important role in the designing of the circuit. The clock signals are important for the operation of a CP circuit. Ideally, clock signals should have zero rise and fall times, constant duty cycles, and zero skew. In reality, clock signals have nonzero skews and nonzero rise and fall times. In practical cases, however, clock signals have nonzero rise and fall times and nonzero skew along with varying duty cycles. The duty cycles can also vary. For a CP circuit, many different clock generator circuits are used. In switched capacitor (S/C) circuits, the clock signals control the switching activities and thereby determine the entire operation of the circuit. No charges are lost when the switching operation is performed. In CP circuits, NOC generator circuit is used to eliminate the clock skew. When the output signals of the NOC signal generator are switching, there must be a time gap between signals so that none of the signals become high. This time gap between signals depends on how much the signals are delayed in the circuit. The propagation delay of the signals depends on the size of the transistor in the NOC generator circuit [11]. A NOC driver is required inside the HVG for optimum pumping facility of the $\mathrm{CP}$ circuit as shown in Fig. 1. While designing high voltage generating $\mathrm{CP}$ circuit, the NOC generator plays an important role in providing the entire anti-phase clock signals for the entire operation of the $\mathrm{CP}$ circuit.

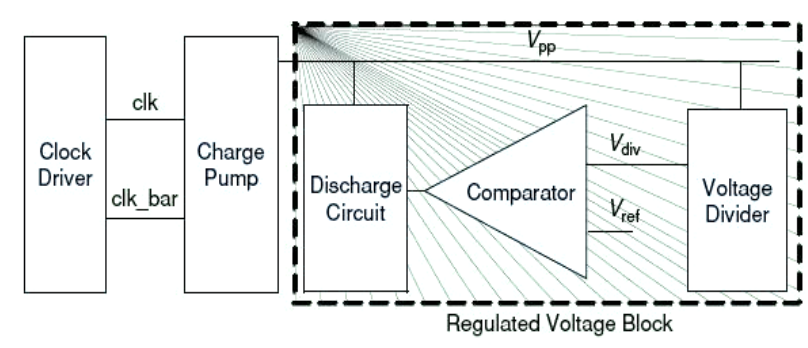

Fig. 1. Block diagram of the HVG.

In this research, a NOC generator is required to produce two anti-phase clock signals for the successful $\mathrm{CP}$ operation 
for the implementation of the HVG. Moreover, this NOC generator will be suitable for the functionality of the low power applications like RFID transponder EEPROM. The designed NOC generator is able to perform faster with lower settling time and dissipates lower power under low power supply voltage. In addition, the NOC generator has been designed with the CEDEC $0.18 \mu \mathrm{m}$ CMOS process.

\section{Methodology}

A NOC generator is required inside the HVG for optimum pumping facility of the $\mathrm{CP}$ circuit. Generally, a clock generator takes a clock signal and produces two-phase NOC signals. Non-overlapping signals are signals operating at the same frequency. None of the non-overlapping signals is high at the time of transition from high to low or vice versa. Generally, a clock generator takes a clock signal and produces two-phase non-overlapping clock. The falling edge of the input passes through the NAND gate, while the rising edge has to propagate first through the other NAND gate and the cascaded delay element. The resulting signals, $\varphi$ and $\varphi_{b}$, have a non-overlapping time equal to the sum of the delay at the NAND gate of the delay element. To construct the delay element, an even number of inverters have been used. When driving long clock lines, additional buffer stages need to be used to maintain sharp output clock rise and fall times [12].

Fig. 2 shows the schematic diagram of the NOC generator. The generated clock pair CLK and CLKB is non-overlapping. In this research, the operation of the clock generator has two parts, where the first part contains four standard cells from "CEDEC Std cells" library 3-x inv01a (INV01A1, INV01A2, and INV01A4) and 1-x nand02a (NAND02A1). In addition, the second part contains three standard cells: 1-x nand02a (NAND02A2) and 2-x inv01a (INV01A5 and INV01A6).

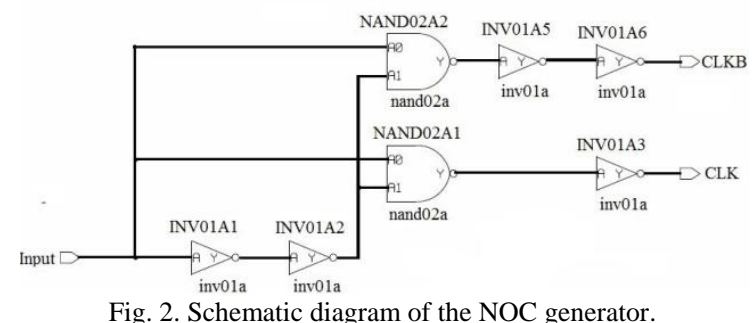

When the falling edge of the input signal is passed through the INV01A1, INV01A2 and NAND02A1 gates, it generates a signal, which is same as the falling edge. This signal again passes through the delay element INV01A4 and produces the signal CLK that is in rising edge. At the same time, the rising edge is propagating first through the other NAND02A2 gate and the cascaded delay element. To construct the delay element, an even number of inverters INV01A5 and INV01A6 is used. After passing through the even number of delay elements, the signal CLKB is generated, which is in the falling edge. Finally, the resulting signals, CLK and CLKB, have a non-overlapping time equal to the sum of the delay at the NAND gates of the delay element.

The NOC generator circuit layout has been designed using the standard cells from the "CEDEC Std cells" library. To design the NOC generator as mentioned in the schematic section, 5-x inv01a, 2-x nand02a are placed side by side as shown in Fig. 3. To reduce the complexity of the design, all logic gates are placed alongside. In addition, this placement is required only one VDD and VSS connection and reduces the complexity of the designs.

Input comes with MET1 from the input A of inv01a gate (marked as 1). The output $\mathrm{Y}$ of this inv01a gate is connected with the input A of the inv01a gate (marked as 2). At the same time, another input from this point is connected with the input $\mathrm{A} 0$ of the nand02a gate (marked as 5). The output $\mathrm{Y}$ of the inv02a (1) is connected with the input A1 of both nand02a gates ( 3 and 5). The output $\mathrm{Y}$ of nand02a gate (3) is added to the input A of inv01a gate (4) through the MET2, which is $0.28 \mu \mathrm{m}$ in width. The output Y of inv01a gate (4) is one of the targeted outputs CLK, which is drawn using the MET3 $(0.28 \mu \mathrm{m})$ layer to avoid the short circuit. The output $\mathrm{Y}$ of nand02a gate (5) is connected to the input $\mathrm{A}$ of inv01a gate (6) and the output $\mathrm{Y}$ is connected with the last inverter inv01a (7). Finally, another targeted output CLKB comes from the output $\mathrm{Y}$ of the inv01a gate (7).

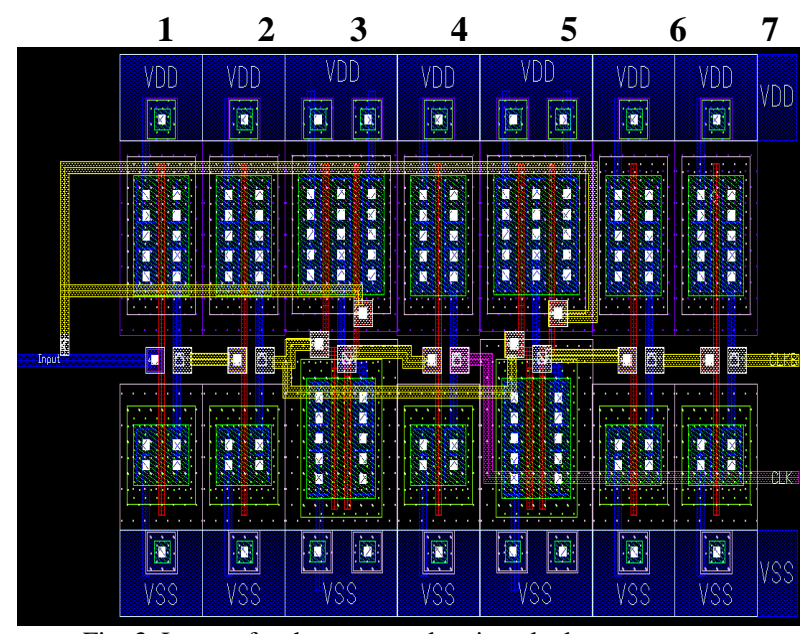

Fig. 3. Layout for the non-overlapping clock generator.

\section{RESUlTS AND DISCUSSION}

The NOC generator circuit is designed in CEDEC $0.18 \mu \mathrm{m}$ CMOS process. The designed NOC circuit has been verified by using the ELDONET simulator of the CEDEC $0.18 \mu \mathrm{m}$ process. Generally, output voltages (CLK and CLKB) are with the same voltage level as the power supply voltage (VDD). Fig. 4 shows the NOC generator test bench, where two output signals are formed to confirm the circuit performance.

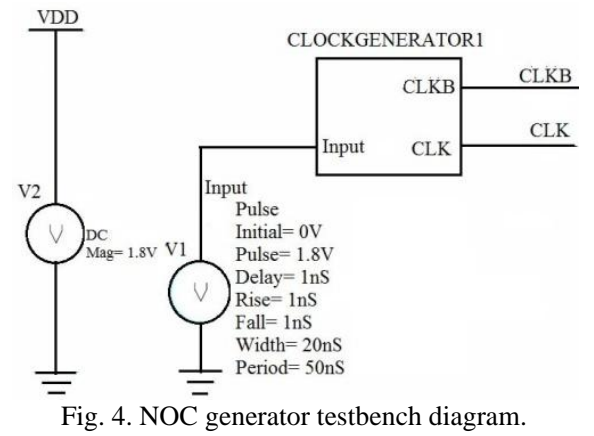

NOC generator circuit is simulated under VDD $=1.8 \mathrm{~V}$. 
From the simulated result as shown in Fig. 5 it is clear that after providing a pulse as an input two anti-phase clock signals CLK and CLKB are generated with the same amplitude of VDD. To design the NOC generator, standard cell inverters and NAND gates are used. The inverters are used to generate delay in the circuit. The required gate delay for the circuit can be selected using the inverters. The delay in the inverters decides the non-overlap period.
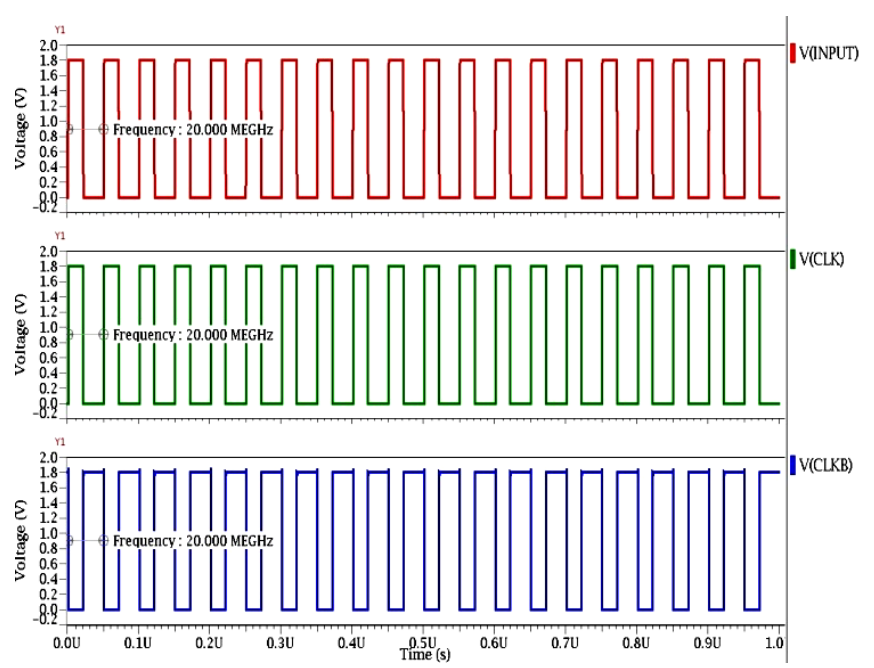

Fig. 5. Simulated results of the NOC generator circuit.

Simulated results for the NOC generator circuit revealed that with the pulse width $21 \mathrm{nS}$ as the input signal, the circuit is able to produce two anti-phase clock signals CLK and CLKB with the pulse width $20.9 \mathrm{nS}$. All the clock signals are produced with the same frequency of $20 \mathrm{MHz}$.

As the focus of this research work is to lower the power dissipation, so the supply voltage for the NOC generation circuit is set to $1.8 \mathrm{~V}$. Similarly, the pulse statement value for the input signal is set to $1.8 \mathrm{~V}$, which is used for initialization. As a result, the output signals CLK and CLKB were generated with the same amplitude level as the supplied input clock signal. The main clock input is running at period $50 \mathrm{nS}$ to yield a clock frequency for the two anti phase clocks of 20 MHz.

Fig. 6 shows the settling time achieved from the simulation of the NOC generator.

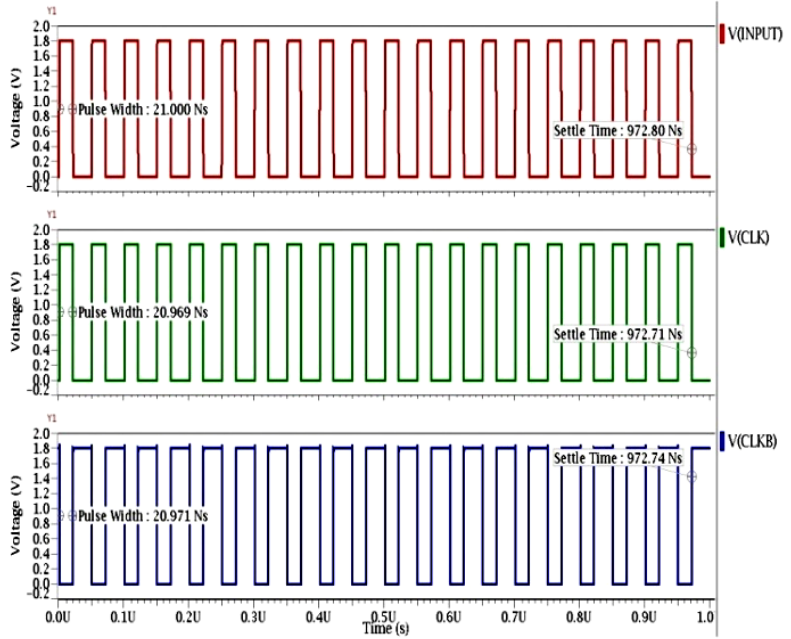

Fig. 6. Simulated results of the NOC generator circuit with settling time.

As the designed NOC generator is a part of readerless
RFID transponder EEPROM, it ought to achieve fast maneuver to pass the clock signals to the $\mathrm{CP}$ circuit of the EEPROM. From the software features, the settling time of the NOC generator is calculated, which is $0.972 \mu \mathrm{S}$ for all the inputs and outputs under 1.8 V VDD, as shown in Fig. 7. Propagation delay between the two clock signals CLK and CLB is also found, which is shown in Fig. 7. From the simulated output it is shown that delay during the rise time between two signals $\mathrm{V}(\mathrm{CLK})$ and $\mathrm{V}(\mathrm{CLKB})$ is achieved $29.05 \mathrm{nS}$, whereas the delay during the fall time is achieved $20.82 \mathrm{nS}$.
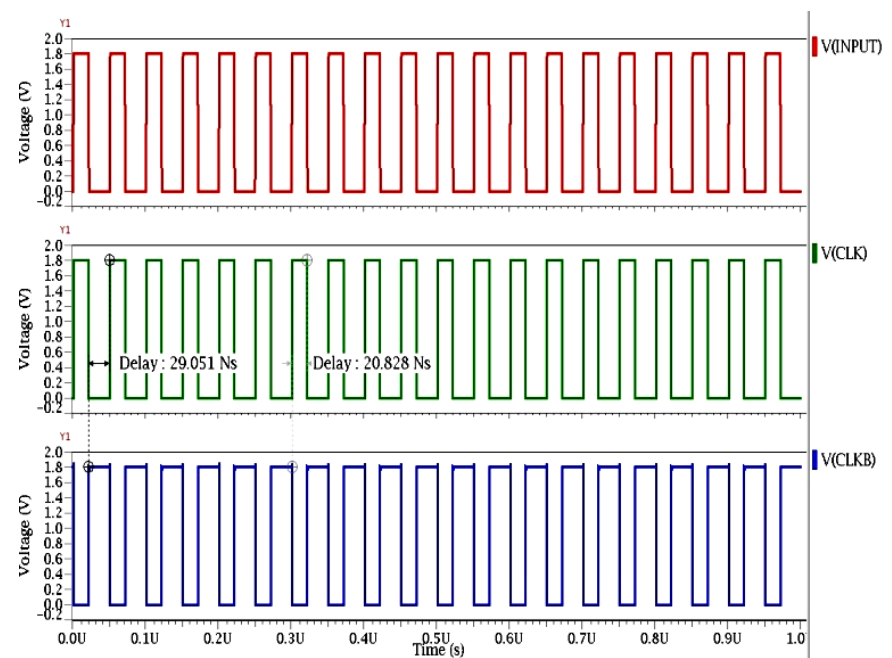

Fig. 7. Simulated results of the NOC generator circuit with rise time and fall time delay.

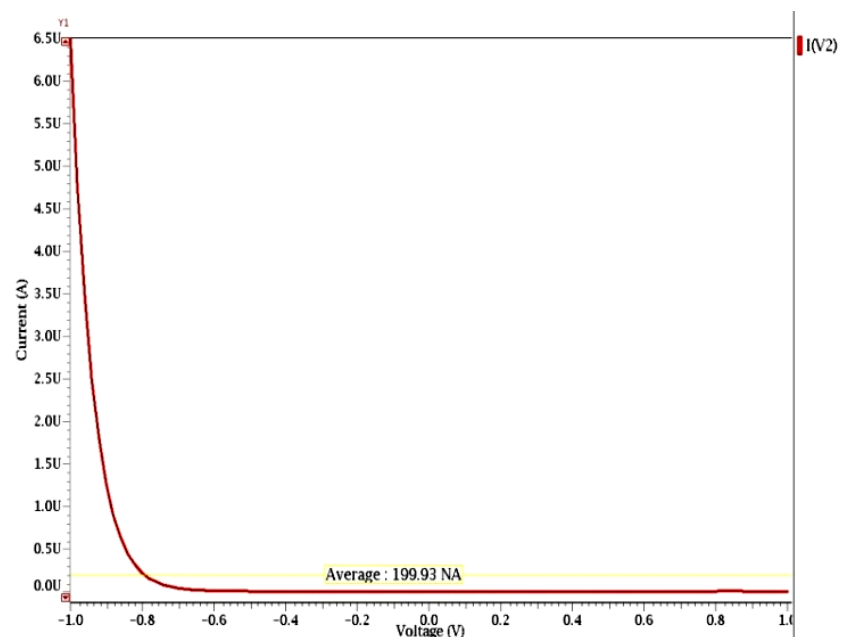

Fig. 8. Simulated results of the NOC generator circuit with average current consumption.

Fig. 8 shows the simulation result of average current consumption of the NOC generator. As the aim of this research work is to minimize the power indulgence, so from the simulation it can be claimed the designed NOC generator is able to restrict the power dissipation, which is found $359.87 \mathrm{nW}$ under the supply voltage $1.8 \mathrm{~V}$.

\section{CONCLUSION}

A NOC generator circuit using delay cells to provide direct clock signals to $\mathrm{CP}$ circuit for HVG is presented in this research. The designed NOC circuit is capable of working in low-voltage power supply. In addition, the output signals require low power dissipation. The simulation results have 
shown that the designed NOC circuit with one input pulse of $20 \mathrm{MHz}$ is able to generate to anti-phase non-overlapping clock signals as the output with $1.8 \mathrm{~V}$ power supply voltage. In addition, the generated output signals are with the same amplitude of VDD, which is compatible with the HVG circuit. Thus, the designed NOC generator will be suitable for the HVG circuit, which is required to generate high voltage internally for NVM storage like RFID transponder EEPROM.

\section{ACKNOWLEDGMENT}

The authors would like to express sincere gratitude to the research grant DLP-2013-016 from the Ministry of Science, Technology and Innovation (MOSTI) and Universiti Kebangsaan Malaysia for supporting this research project.

\section{REFERENCES}

[1] M. J. Uddin, M. I. Ibrahimy, M. B. I. Reaz, and A. N. Nordin, "Design and application of radio frequency identification systems," European Journal of Scientific Research, vol. 33, no. 3, pp. 438-453, 2009.

[2] F. M. Yasin, M. K. Khaw, and M. B. I. Reaz, "Radio Frequency Identification: Evolution of Transponder Circuit Design," Microwave Journal, vol. 49, no. 6, p. 56, 2006.

[3] K. Ohsaki, N. Asamoto, and S. Takagaki, "A single poly EEPROM cell structure for use in standard CMOS processes," IEEE Journal of Solid-State Circuits, vol. 29, no. 3, pp. 311-316, Mar. 1994.

[4] Z. Dixian, Y. Na, X. Wen, Y. Liwu, W. Junyu, and M. Hao, "A low-power, single-poly, non-volatile memory for passive RFID tags," Chinese Journal of Semiconductors, vol. 29, pp. 99-104, Jan. 2008.

[5] B. Wang, H. Nguyen, Y. Ma, and R. Paulsen, "Highly reliable 90-nm logic multitime programmable NVM cells using novel work-function-engineered tunneling devices," IEEE Transactions on Electron Devices, vol. 54, no. 9, pp. 2526-2530, Sept. 2007.

[6] L. Rahman, M. B. I. Reaz, C. T. Gyu, and M. Marufuzzaman, "Design of sense amplifier for non volatile memory," Revue Roumaine Des Sciences Techniques, vol. 58, no. 2, pp. 173-182, 2013.

[7] J. Raszka, M. Advani, V. Tiwari, L. Varisco, N. D. Hacobian, A. Mittal, M. Han, A. Shirdel, and A. Shubat, "Embedded flash memory for security applications in a $0.13 \mu \mathrm{m}$ CMOS logic process," in Proc. International Solid-State Circuits Conference (ISSCC), IEEE, 15-19 Feb., 2004 .

[8] L. F. Rahman, M. B. I. Reaz, M. A. M. Ali, and M. Marufuzzaman, "Implementation of sense amplifier in $0.18-\mu \mathrm{m}$ CMOS process," Electronics and Electrical Engineering, vol. 120, no. 4, pp. 113-116, 2012.

[9] M. G. Mohammad, M.-J. Ahmad, and M. B. Al-Bakheet, "Switched positive/negative charge pump design using standard CMOS transistors," IET Circuits Devices System, vol. 4, no. 1, pp. 57-66, Jan. 2010.

[10] IEEE Standard Definitions and Characterization of Floating Gate Semiconductor Arrays, IEEE Std. 1005, 1999.

[11] F. Kavak, "A sizing algorithm for non-overlapping clock signal generators," Master thesis, Institute of Technology, Linköping University, Sweden. Jun. 2004

[12] F. Pan and T. Samaddar, Charge Pump Circuit Design, New York: McGraw-Hill, 2006.

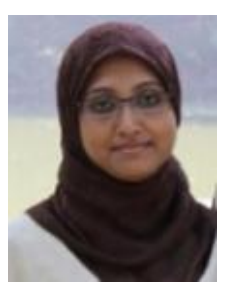

Labonnah F. Rahman was born in Bangladesh in September 1983. She received her M.Sc. degree in electrical, electronic and systems engineering from Universiti Kebangsaan Malaysia, UKM Bangi, Malaysia in 2012. Since 2007 to 2009, she worked for GlaxoSmithKline Bangladesh Limited as an IT systems executive. Currently she is doing her Ph.D. by research in the area of IPv6 based RFID systems under the Dept. of Electrical, Electronic and Systems Engineering in UKM. Her research interests are in the following fields: IP networking, wireless networking, data communication, microchip design and fabrication.

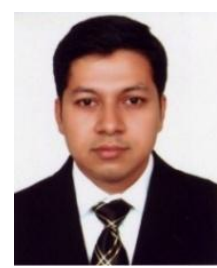

M. B. I. Reaz was born in Bangladesh in December 1963. He received his B.Sc. and M.Sc. degrees in applied physics and electronics, both from University of Rajhashi, Bangladesh, in 1985 and 1986, respectively. He received his D.Eng. degree in 2007 from Ibaraki University, Japan. He is currently a professor in the Department of Electrical, Electronic and Systems Engineering, Universiti Kebangsaan Malaysia, Malaysia involving in teaching, research and industrial consultation. He is a regular associate of the Abdus Salam International Center for Theoretical Physics since 2008. He is also a senior member of IEEE. He has vast research experiences in Norway, Ireland and Malaysia. He has published extensively in the area of IC design and biomedical application IC. He is author and co-author of more than 200 research articles in design automation and IC design for biomedical applications.

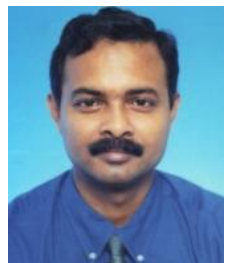

Mohd. Marufuzzaman was born in Bangladesh in June 1983. He received the M.Sc. degree in electrical, electronic and systems engineering from Universit Kebangsaan Malaysia, UKM Bangi, Malaysia in 2012. Currently he is doing his Ph.D. in the Dept. of Electrical, Electronic and Systems Engineering in University Kebangsaan Malaysia. His research interests are in the following fields: IP networking, design and fabrication. 